\title{
Chuvash erythrocytosis
}

INSERM

\section{Source}

INSERM. (1999). Orphanet: an online rare disease and orphan drug data base. Chuvash erythrocytosis. ORPHA:238557

Chuvash erythrocytosis is a rare, genetic, congenital secondary polycythemia disorder characterized by increased hemoglobin, hematocrit and erythropoietin serum levels and normal oxygen affinity, which usually manifests with headache, dizziness, dyspnea and/or plethora. Patients present an increased risk of hemorrhage, thrombosis and early death. 